\title{
Procedure Agent Sponsor Identifier
}

National Cancer Institute

\section{Source}

National Cancer Institute. Procedure Agent Sponsor Identifier. NCI Thesaurus. Code C162118.

One or more sponsor defined characters used to identify, name, or characterize the procedure agents. 